\title{
Effects of percutaneous neuromodulation on the mechanical characteristics of the rectus femoris and the vastus lateralis in football players: a pilot study
}

\author{
Padrón-Benítez A. ${ }^{1}$ Rodríguez-Ruiz D. ${ }^{1,2}$ Ortega-Puebla F. ${ }^{3}$ Valdesuso-Domínguez R. ${ }^{3}$ \\ ${ }^{1}$ Fix\&Fit, Centro de Fisioterapia, Readaptación y Entrenamiento, Las \\ Palmas de Gran Canaria, Spain \\ 2 Department of Physical Education, Universidad de Las Palmas de \\ Gran Canaria, Spain \\ ${ }^{3}$ Clínica de Rehabilitación Avanzada Forehav, Elche, Spain
}

Rev Fisioter Invasiva 2019;2:86-87.

\begin{abstract}
Keywords

- percutaneous electrical nerve stimulation (PENS)

- myography

- quadriceps muscle

- femoral nerve

- soccer

Background and Aims Percutaneous neuromodulation is an ultrasound-guided technique aimed at the peripheral nerve, using an acupuncture needle as an electrode for a TENS type current which has demonstrated to have positive effects for pain but not for muscle activity. Tensiomyography (TMG) is a non-invasive assessment technique, which is reliable and provides instant information on the mechanical characteristics of superficial muscles. The aim of this study is to evaluate whether percutaneous neuromodulation produces effects on muscle contraction parameters.

Material and Methods A longitudinal, analytic and experimental study with a control group, comprising a sample of 11 football players in the 3rd Division, randomly assigned to an intervention group $(I G=5)$ and a control group $(C G=6)$. Athletes were assessed using tensiomyography (TMG-S2, EMF-FURLAN \& Co.) parameters: maximum deformation $(\mathrm{Dm})$, response time $(\mathrm{Td})$, contraction time $(\mathrm{Tc})$, relaxation time $(\mathrm{Tr})$ and time of contraction maintenance (Ts) of the rectus femoris (RF) and vastus lateralis (VL). Both legs were assessed immediately after the training session (Pre), after 10' rest period post intervention (Post 1 ) and after 12 hours of rest on the following day (Post2). Both femoral nerves were intervened with a needle on the long axis and in a transverse section just below the same. An Electrostimulator (ITO, model ES-160), was applied to the IG during 5' using a TENS current of $200 \mu \mathrm{s}, 1 \mathrm{~Hz}$ and with sufficient intensity to achieve a visible contraction of the quadriceps without discomfort. The channel was closed with adhesive electrodes placed medial to the ASIS. The CG received a placebo treatment with needles and electrodes placed on the same anatomical points and connected to an electrostimulator without current during 5' (Physio Invasiva device). Results On the right RF the Td decreased in the IG (pre/post2: $P=0.005$ ) and between groups (post1: $P=0.017$ and post2: $P=0.014$ ). For $T c$ we found differences between the IG (pre/post2: $P=0.008$ ) and the CG (post1/post2: $P=0.019$ ). The left RF increased TC in the CG $(P=0.036)$ and pre/post 2 and Ts in the CG compared to the IG (post2: $P=0.0480 .048$ ). In the left VL Dm increased (pre/post2: $P=0.046$ and post1/ post2: $\mathrm{P}=0.018), \mathrm{Td}$ (pre/post2: $\mathrm{P}=0.010$ ) and Tc (pre/post2: $\mathrm{P}=0.051$ and post $1 /$ post2: $\mathrm{P}=0.003)$ for the $C G$.
\end{abstract}

DOI https://doi.org/ $10.1055 / \mathrm{s}-0039-3401867$. ISSN 2386-4591.
Copyright $\odot 2019$ by Thieme Revinter Publicações Ltda, Rio de Janeiro, Brazil
License terms

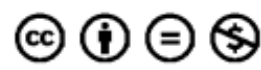


In the IG, the right $V L$, both $T d(P=0.024)$ and $T c(P=0.021)$ decreased between post1/post2.

For the intragroup analysis (pre, post1 and post2) the Student's t-test was used, whereas for the intragroup analysis, the ANOVA was used (significance $>P$ 0.05).

Conclusions Considering the characteristics of the sample and understanding that the decrease of TMG parameters represents an improvement in the mechanical characteristics of the muscle, the findings of this pilot study suggest that the intervention with percutaneous neuromodulation in the IG produces greater recovery and improved activation after 12 hours on the RF and VL. However, the increase of these parameters in the CG results in greater fatigue. 